\title{
Allopolyploid origin in Rubus (Rosaceae) inferred from nuclear granule-bound starch synthase I (GBSSI) sequences
}

Yan Wang ${ }^{1,2+}$, Qing Chen ${ }^{2+}$, Tao Chen², Jing Zhang ${ }^{1,2}$, Wen He², Lin Liư ${ }^{3}$ Ya Luo ${ }^{2}$, Bo Sun ${ }^{2}$, Yong Zhang ${ }^{2}$, Hao-ru Tang ${ }^{1,2}$ and Xiao-rong Wang ${ }^{1,2^{*}}$

\begin{abstract}
Background: Polyploidy and hybridization are ubiquitous in Rubus L., a large and taxonomically challenging genus. Chinese Rubus are mainly concentrated into two major sections, the diploid Idaeobatus and the polyploid Malachobatus. However, it remains unclear to be auto- or allo- polyploid origin of polyploids in Rubus. We investigated the homoeologs and the structure of the GBSSI-1 (granule-bound starch synthase I) gene in 140 Rubus individuals representing 102 taxa in 17 (out of the total 24) subsections of 7 (total of 12) sections at different ploidy levels.

Results: Based on the gene structure and sequence divergence, we defined three gene variants, GBSSI-1a, GBSSI-1b, and GBSSI-1c. When compared with GBSSI-1a, both GBSSI-1b and GBSSI-1c have a shorter fourth intron, and GBSSI-1c had an additional deletion in the fifth intron. For diploids, either GBSSI-1a or GBSSI-1b was detected in 56 taxa consisting of 82 individuals from sect. Idaeobatus, while both alleles existed in $R$. pentagonus and R. peltatus. Both homoeologs GBSSI-1a and GBSSI-1b were identified in 39 taxa (48 individuals) of Malachobatus polyploids. They were also observed in two sect. Dalibardastrum taxa, in one sect. Chamaebatus taxon, and in three taxa from sect. Cylactis. Interestingly, all three homoeologs were observed in the three tetraploid taxa. Phylogenetic trees and networks suggested two clades (I and II), corresponding to GBSSI-1a, and GBSSI-1b/1c sequences, respectively. GBSSI-1 homoeologs from the same polyploid individual were resolved in different well-supported clades, and some of these homoelogs were more closely related to homoelogs in other species than they were to each other. This implied that the homoeologs of these polyploids were donated by different ancestral taxa, indicating their allopolyploid origin. Two kinds of diploids hybridized to form most allotetraploid species. The early-divergent diploid species with GBSSI-1a or -1b emerged before polyploid formation in the evolutionary history of Rubus.
\end{abstract}

Conclusion: This study provided new insights into allopolyploid origin and evolution from diploid to polyploid within the genus Rubus at the molecular phylogenetic level, consistent with the taxonomic treatment by Yü et al. and Lu.

Keywords: Rubus, GBSSI-1, Section Idaeobatus, Sect. Malachobatus, Allopolyploid origin, Hybridization, Evolution

\footnotetext{
* Correspondence: wangxr@sicau.edu.cn

†Yan Wang and Qing Chen contributed equally to this work.

${ }^{1}$ Institute of Pomology and Olericulture, Sichuan Agricultural University, Chengdu, China

${ }^{2}$ College of Horticulture, Sichuan Agricultural University, Chengdu, China

Full list of author information is available at the end of the article
}

(c) The Author(s). 2019 Open Access This article is distributed under the terms of the Creative Commons Attribution 4.0 International License (http://creativecommons.org/licenses/by/4.0/), which permits unrestricted use, distribution, and reproduction in any medium, provided you give appropriate credit to the original author(s) and the source, provide a link to the Creative Commons license, and indicate if changes were made. The Creative Commons Public Domain Dedication waiver (http://creativecommons.org/publicdomain/zero/1.0/) applies to the data made available in this article, unless otherwise stated. 


\section{Background}

The genus Rubus L. belongs to the subfamily Rosoideae of the family Rosaceae, with 750-1000 species distributed worldwide except Antarctica [1-3]. Focke [1-3] established the widely adopted Rubus taxonomy that contained 12 subgenera, with the three largest subgenera of Idaeobatus, Malachobatus, and Rubus (Additional file 1). The number of Rubus species in China accounts for 97\% of the total in Asia. More than 200 species have been recorded in China, of which 139 species are indigenous [4]. Basing upon the evolutionary tendency of morphological features, chromosome numbers of certain species and the distribution patterns of species, taxonomists in China [4-6] proposed a new systematic arrangement of Chinese Rubus, with eight sections (Additional file 1). The two taxonomic systems are concordant in the classification of most species, while the arrangement of sections is presented in a reverse order to those of Focke's system (Additional file 1). Most species are assigned into two major sections, Idaeobatus and Malachobatus, including 11 and 13 subsections, respectively [5]. Section Idaeobatus is characterized by its shrub habit armed with sharp prickles, aciculae or setae, leaves pinnately compound or simple, stipules attached to the petioles, flowers hermaphroditic and often in terminal or axillary inflorescences, very rarely solitary, and drupelets separating from the receptacles $[5,6]$. In contrast, members of sect. Malachobatus are usually woody with prickles, simpleleaved, stipules free, flowers bisexual and in cymose panicles, subracemes, and drupelets adhering to receptacles $[5,6]$.

The evolutionary history of Rubus species inferred from different analyses has been argued for a long time. Based on morphological and chromosomal data, $\mathrm{Lu}$ [4] suggested that evolution in Rubus proceeded from woody to herbaceous plants, and from species with compound leaves to simple leaves. This proposal was consistent with the view of Kalkman [7]. However, ITS data conflicted with these hypotheses: primarily semi-herbaceous, simpleleaved species occupied early-diverging positions in the trees [8].

Polyploidy and hybridization are common in Rubus [9]. Species of sects. Idaeobatus are predominantly diploids $(2 n=2 x=14)$, while sects. Malachobatus, Dalibardastrum, and Chamaemorus are exclusively polyploids $(2 n=4 x, 6 x, 8 x, 14 x=28,42,56,84)$ [9-11]. In addition, interspecific hybridization and facultative apomixis play an important role in sect. Rubus, which blurred species boundaries [9]. Based on chromosomal karyotype, meiotic pairing and fluorescence in situ hybridization (FISH) analyses, several polyploids from sect. Malachobatus have been demonstrated to be of allopolyploid origin $[12,13]$. Hybridization in Rubus occurs not only between closely related species from the same section [14-21], but also between species from different sections
[22, 23]. Soltis \& Soltis [24] proposed that, allopolyploid formation via interspecific hybridization and subsequent genome doubling has become an important mode of speciation in higher plants. Therefore, based on the assumption and our previous studies [12, 13, 25], we speculated the majority of polyploids being of allopolyploid origin. It is needed to be further elucidated by powerful evidence.

To reconstruct the evolutionary history of plant polyploid species using molecular data, it is necessary to deal with the presence and the evolutionary fate of multiple gene copies resulting from paralogs and orthologs [26]. Identification of homoeologs in polyploids is crucial for reliable phylogeny reconstruction, and also informative for identifying parental lineages and inferring auto- or allo- formation of polyploids [27]. Low-copy nuclear genes that succeeded in other Rosaceae are potentially ideal nuclear markers for phylogenetic analysis of Rubus complex. The GBSSI gene, coding for granule-bound starch synthase I, is single copy in most diploid angiosperms [28]. The entire gene consists of 13 translated exons and 12 introns. Phylogenetic studies have shown that GBSSI exons and introns are useful in resolving relationships among closely related genera and species [26], especially in detecting ancient hybridization events of polyploids [29, 30]. In Rubus and most Rosaceae, the GBSSI gene is represented by two paralogous loci, GBSSI-1 and GBSSI-2, which can be differentiated by specific indels [29, 31]. Partial GBSSI-2 sequences, as a single copy gene, have provided high phylogenetic resolution within Rubus [25]. Additionally, two different alleles of GBSSI-1 were detected in octoploid R. chamaemorus, inferring it to be an ancient allopolyploid that resulted from multiple hybridization events [30]. It is believed that GBSSI-1 gene is extremely helpful to reveal the origin and evolution for Rubus polyploids.

In this study, we explored the utility of GBSSI-1 to elucidate the evolutionary history of genus Rubus and particularly the auto- or allo- polyploid origin of the polyploids. Our objectives were (i) to investigate the number of GBSSI-1 variants within Rubus at different ploidy levels, (ii) to analyze the gene structure and conduct homoeolog identification, and (iii) to provide new insights into the polyploid origin and evolutionary history within Rubus by reconstructing the phylogeny.

\section{Results \\ Gene variants and orthology identification of GBSSI-1 within Rubus}

As shown in Fig. 1 and Additional file 2, we obtained different GBSSI-1 variants (GBSSI-1a, GBSSI-1b and GBSSI-1c) within Rubus at different ploidy levels. Based on the definition of ortholog by Yu et al. [32], we carried out the orthology assessment. The different GBSSI-1 variants shared $>90 \%$ identity at the amino acid sequence 


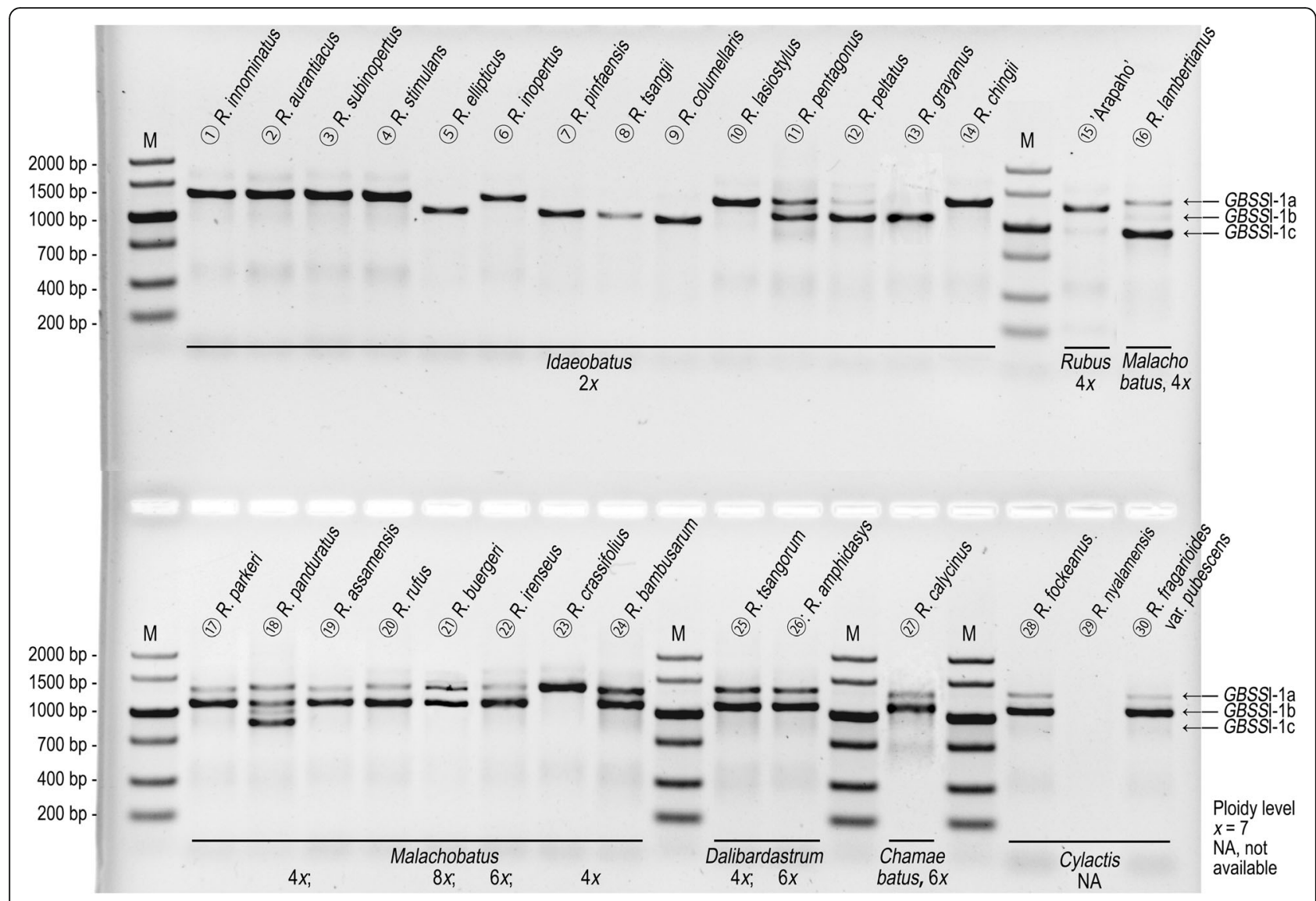

Fig. 1 The number of GBSSI-1 variants within Chinese Rubus at different ploidy levels. The arrows represent the positions of individual GBSSI-1 variants

level with a significant E-value $\left(<10^{-10}\right)$, and distributed on the same zone of chromosome 7 by alignment with reference genome of diploid $R$. occidentalis $\mathrm{L}$. [33] (Additional file 3). Orthology of the Rubus diploid sequences was also assessed using phylogenetic analysis. The dataset was obtained from our GBSSI-1 sequences from Rubus diploids and from GBSSI (1 and 2) coding region sequences of Rosaceae species available in GenBank. This matrix included 378 nucleotides sites, of which 141 were constant and 164 were phylogenetically informative. The phylogenetic tree (Fig. 2) grouped all the Rosaceae GBSSI sequences into two well-supported clades with bootstrap values of 96 and 95\%, respectively. These clades represented paralogous genes, corresponding to GBSSI-1 and GBSSI-2 according to Evans et al. [29]. In the GBSSI1 clade, all the Rubus diploid sequences fell in a wellsupported clade (99\% BS), which provided evidence that these sequences were orthologous.

For diploids, GBSSI-1a was detected in species of subsections Thyrsidaei, Idaeanthi, Pileati, and Wushanenses, and Corchorifolii, and most Stimulantes and Pungentes species (Fig. 1, (1)-(4), (6), (10, (14)), while GBSSI$1 \mathrm{~b}$ was detected in subsects. Rosaefolii, Leucanthi, and Corchorifolii (Fig. 1, (8), (9, (13), as well as R. ellipticus of subsect. Stimulantes, and $R$. pinfaensis, $R$. macilentus and R. simplex of subsect. Pungentes of sect. Idaeobatus (Fig. 1, (5), (7)). Both GBSSI-1a and GBSSI-1b alleles were found in subsects. Alepestres and Peltati species (Fig. 1, (111, (12). Genotyping patterns varied among polyploids. Only one copy was observed in blackberry cultivar 'Arapaho' (4x) of sect. Rubus (Fig. 1, (15)). Both GBSSI-1a and GBSSI-1b homoeologs were detected in polyploids including tetraploids, hexaploid, and octoploid of sect. Malachobatus (Fig. 1, (16, (17), (19-(2), (2)). R. panduratus had three alleles, GBSSI-1a, GBSSI-1b and GBSSI-1c (Fig. 1, (18), and R. crassifolius possessed only GBSSI-1a (sequence not obtained) (Fig. 1, (3)). There were two homoeologs (GBSSI-1a and -1b) in sects. Dalibardastrum, Chamaebatus, and Cylactis species (Fig. 1, (2)-(3), band of $R$. nyalamensis not shown).

\section{Gene structure and sequence characteristics}

According to the gene structure and sequence divergence, three homoeologs representing GBSSI-1a, GBSSI1 b, and GBSSI-1c were identified (Fig. 3). GBSSI-1a (e.g., from $R$. odoratus, GenBank no. AF285994), had a classical GBSSI gene structure with eight introns (part of the full-length sequence). Similar structure was observed in 


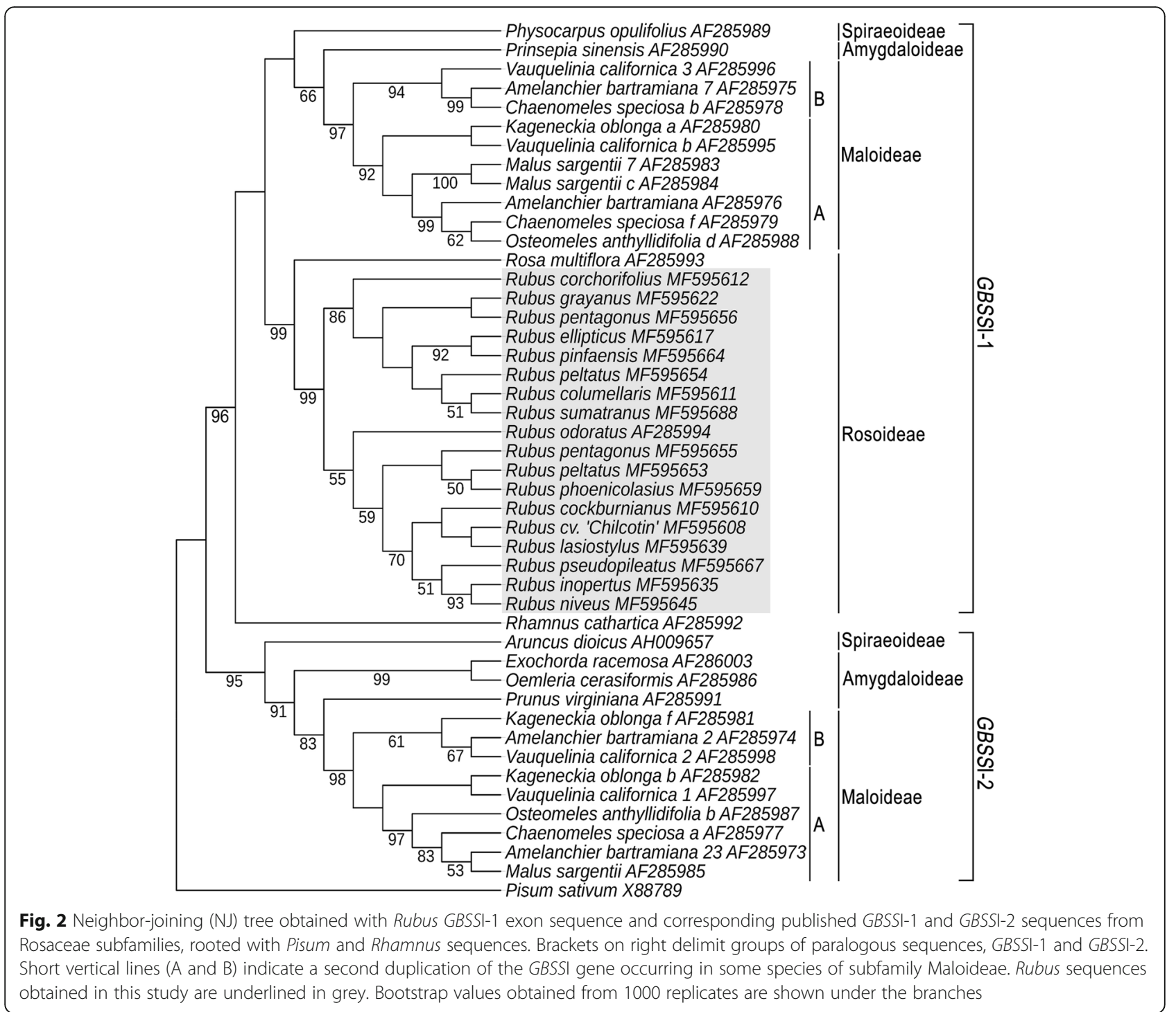

GBSSI-1b and GBSSI-1c, but intron length varied between and within GBSSI-1a, $-1 \mathrm{~b}$ and $-1 \mathrm{c}$. The intron 4 of GBSSI-1b and GBSSI-1c was at least 260 bp shorter than GBSSI-1a. An additional missing intron 5 was detected in GBSSI-1c (Fig. 3, a-c). In addition, a longer 4th intron in Rosoideae GBSSI-1 (Fig. 3, a-d) was observed than other three subfamilies (Fig. 3, e-g), consistent with the results of Evans et al. [29].

After treating the gaps as missing data, we obtained 195 sequences for GBSSI-1 gene (Table 1). GBSSI-1a existd in 83 individuals whereas GBSSI-1b was found in 58 individuals. Three taxa containing five individuals possess GBSSI-1c. The final aligned GBSSI-1a consisted of 1296 nucleotides with length ranging from 1139 to 1234 base pairs. There were 441 (34.03\%) variable characters, of which 257 (19.83\%) were parsimony-informative. The aligned intron 4 was composed of $517 \mathrm{bp}$ with length ranging from 403 to $484 \mathrm{bp}$, which had 188 variable sites. Seven indels were present in the entire gene alignment. The indels consisted of 1-303 nucleotides. Two relatively large ones (an insertion of $136 \mathrm{bp}$, and an insertion of $303 \mathrm{bp}$ ) were found in GBSSI-1a group.

The length of GBSSI-1b varied from 942 to 1001 bases. There were 234 (22.76\%) variable sites, of which 134 (13.04\%) were parsimony-informative in 1028 aligned nucleotides. The intron 4 contained 252 aligned nucleotides from 191 to $249 \mathrm{bp}$, and 65 variable sites. The alignment of the entire gene had four indels, each including 1 to 9 nucleotides. The aligned GBSSI-1c contained $913 \mathrm{bp}$ with length range from 760 to $822 \mathrm{bp}$, of which just 11 were variable. JModelTest suggested that the best-fit model selected by Akaike Information Criterion (AIC) was TIM2 + G for GBSSI-1 dataset.

\section{Phylogenetic analysis}

The GBSSI-1 gene tree generated by both Maximum Likelihood (ML) and Bayesian Inference (BI) analyses 


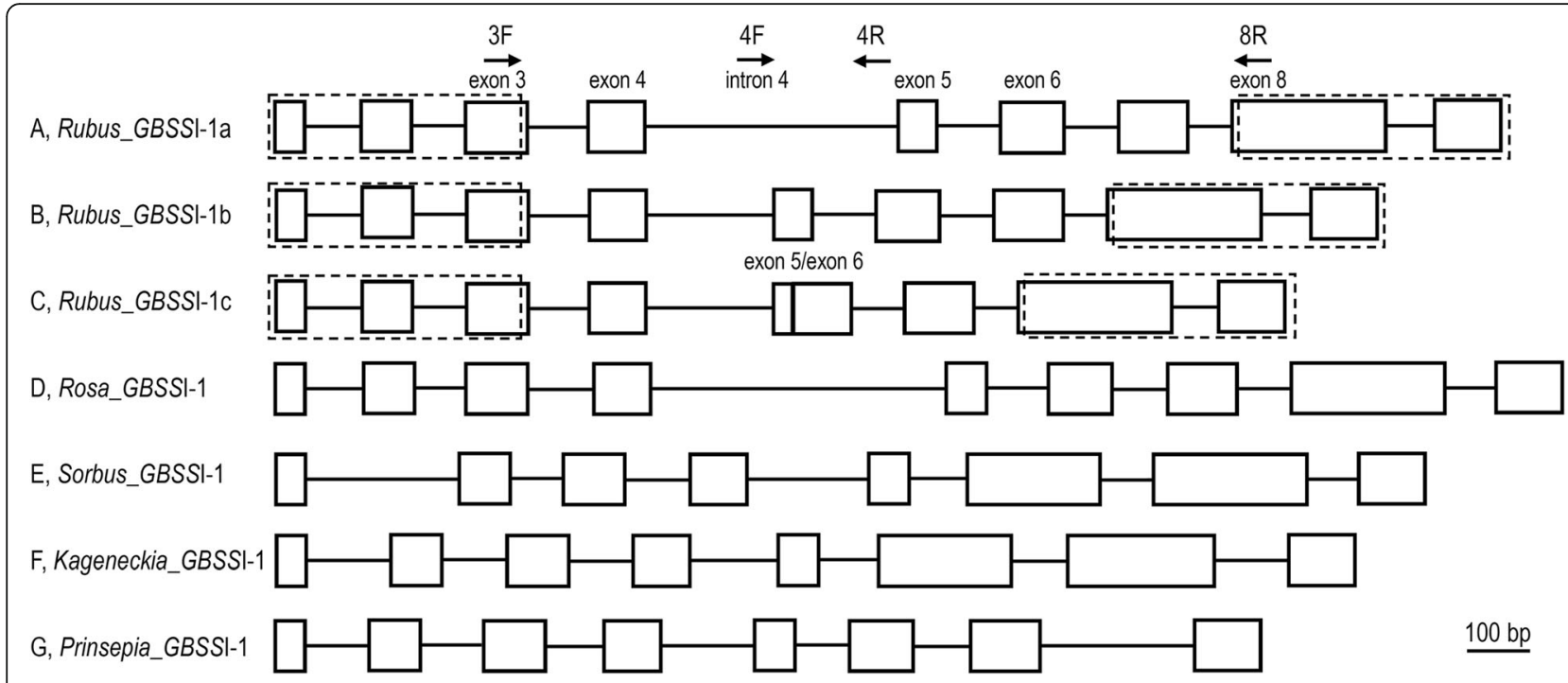

Fig. 3 Schematic diagram of GBSSI-1 gene within the family Rosaceae. Open boxes represent exons, and connecting lines represent introns. The fourth intron of GBSSI-1b and GBSSI-1c was at least 260 bp shorter than GBSSI-1a, and an additional missing fifth intron was detected in GBSSI-1c. Arrows represent the locations and directions of primers used for PCR amplification. Rows A, B and C are different GBSSI-1 genes from Rubus obtained in this study; Rows D-G are GBSSI-1 sequences from Rosa (Rosoideae, AF285993), Sorbus (Maloideae, AF500468), Kageneckia (Spiraeoideae, DQ874892), and Prinsepia (Amygdaloideae, AF285990). The dashed box represents the fragment not obtained in this study

resulted in largely congruent tree topologies, suggesting two major lineages within Rubus (Figs. 4, 5, Additional files 4, 5). Clade I consisted of four subclades (A-D), corresponding to most taxa with GBSSI-1a. As shown in Fig. 4, subclades A and B were represented by $R$. odoratus of sect. Anoplobatus, $R$. fragarioides var. pubescens of sect. Cylactis and four sect. Idaeobatus species. All samples of sect. Malachobatus, and sect. Dalibardastrum, as well as $R$. peltatus of subsect. Peltati from sect. Idaeobatus formed a monophyletic group (C1) with high support values (86\% BS, 1.00 PP). Rubus fockeanus (C2) from sect. Cylactis, R. calycinus (C3) from sect. Chamaebatus, and $R$. pentagonus (C4) from subsect. Alpestres of sect. Idaeobatus (C4) and $\mathrm{C} 1$ were sister to each other. The four groups formed a well-supported $(84 \%$ BS, 1.00 PP) subclade C. Subclade D included species of subsections Thyrsidaei, Idaeanthi, Pileati, and Wushanenses, and most Stimulantes and Pungentes from sect. Idaeobatus without clear circumscription among subsections based on traditional taxonomy (0.76 PP). Blackberry cultivar 'Arapaho' of sect. Rubus was nested within the subclade D.

Clade II was divided into six subclades (E-J), corresponding to all taxa with GBSSI-1b/1c as well as four taxa with GBSSI-1a (Fig. 5). The remaining sect. Idaeobatus species were mainly clustered into four subclades (E, G, H, and I). The subsect. Corchorifolii taxa dispersed in the two groups $\mathrm{E}$ and H1 with GBSSI-1a and -1b, respectively. Group $\mathrm{H} 2$ consisted of $R$. ellipticus from subsect. Stimulantes and R. pinfaensis of subsect. Pungentes. Subclade G corresponded to subsect. Rosaefolii species (68\% BS, 1.00 PP). Subsect. Leucanthi species and $R$.

Table 1 Sequence variations of GBSSI-1 homoeologs in Rubus (excluding outgroups)

\begin{tabular}{|c|c|c|c|c|c|c|}
\hline Region & $\begin{array}{l}\text { Number of } \\
\text { individuals }\end{array}$ & $\begin{array}{l}\text { Number of } \\
\text { sequences }\end{array}$ & $\begin{array}{l}\text { Length } \\
\text { range (bp) }\end{array}$ & $\begin{array}{l}\text { Aligned nucleotide } \\
\text { length (bp) }\end{array}$ & Variable sites (\%) & $\begin{array}{l}\text { Parsimony informative } \\
\text { sites (\%) }\end{array}$ \\
\hline GBSSI-1 & 140 & 195 & $760-1234$ & 1325 & $583(44.00 \%)$ & $366(27.62 \%)$ \\
\hline GBSSI-1 intron & 140 & 195 & $482-858$ & 938 & $436(46.48 \%)$ & $273(29.10 \%)$ \\
\hline GBSSI-1a & 83 & 118 & 1139-1234 & 1296 & 441 (34.03\%) & $257(19.83 \%)$ \\
\hline GBSSI-1a intron & 83 & 118 & $763-858$ & 917 & $340(37.08 \%)$ & 192 (20.94\%) \\
\hline GBSSI-1a intron 4 & 83 & 118 & $403-484$ & 517 & 188 (36.36\%) & $106(20.50 \%)$ \\
\hline GBSSI-1b & 58 & 72 & $942-1001$ & 1028 & $234(22.76 \%)$ & $134(13.04 \%)$ \\
\hline GBSSI-1b intron & 58 & 72 & $563-621$ & 641 & 170 (26.52\%) & 98 (15.29\%) \\
\hline GBSSI-1b intron 4 & 58 & 72 & $191-249$ & 252 & 65 (25.79\%) & 34 (13.49\%) \\
\hline GBSSI-1C & 5 & 5 & $760-822$ & 913 & 11 (1.20\%) & - \\
\hline
\end{tabular}




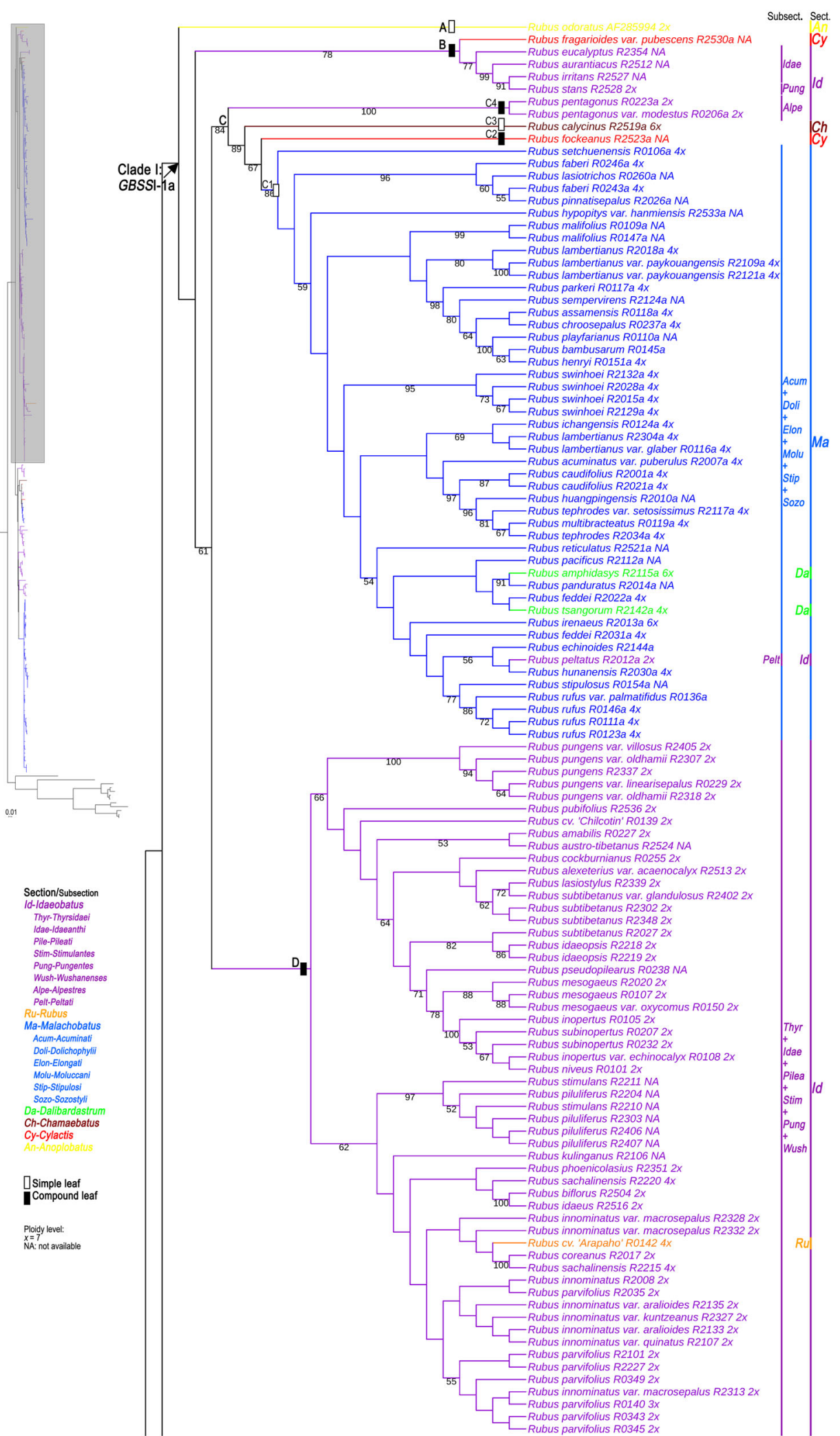

Fig. 4 Maximum likelihood (ML) tree inferred from the GBSSI-1a sequences of Rubus. Bootstrap values $>50$ based on 1000 replicates are provided below the branches 


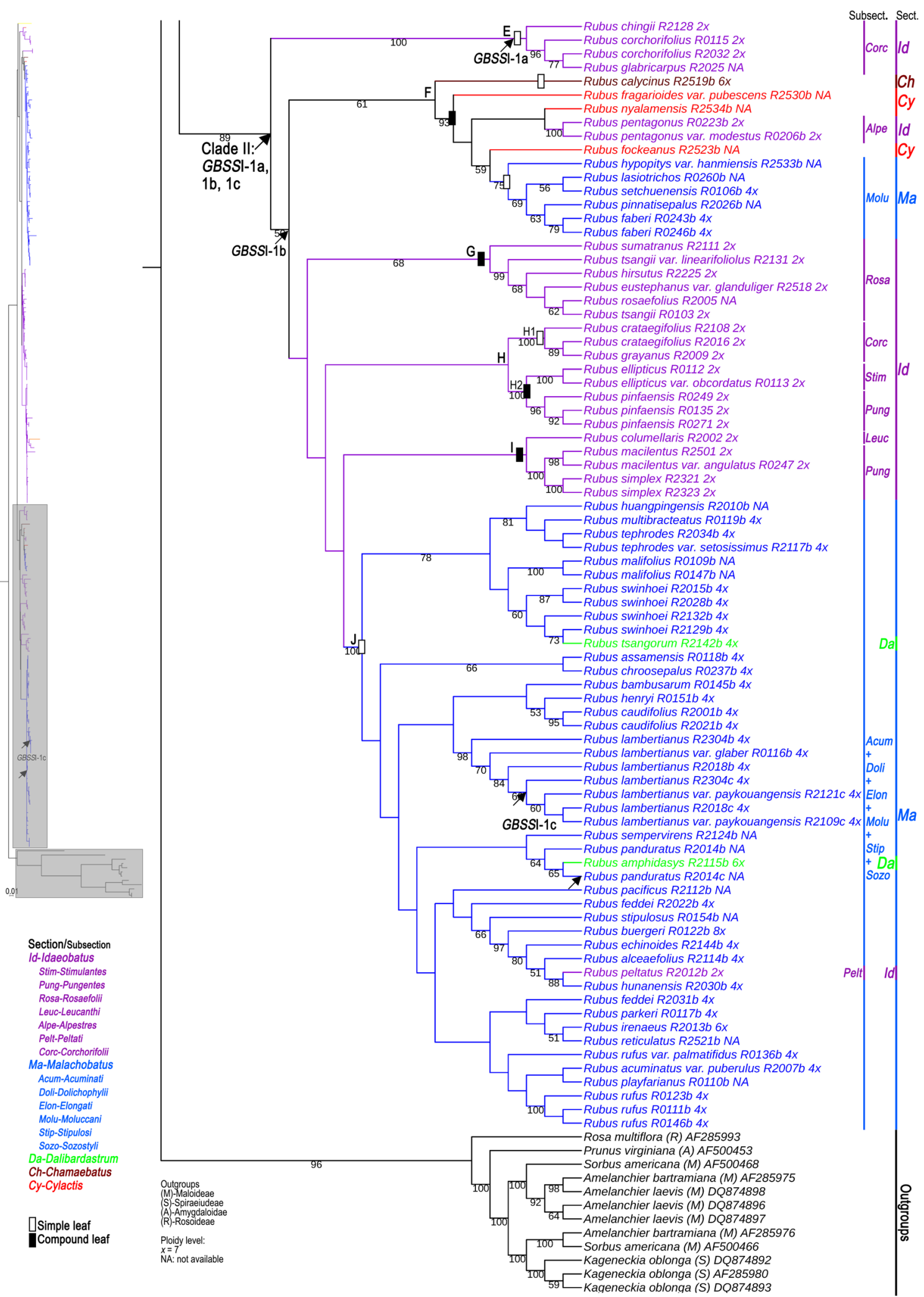

Fig. 5 Maximum likelihood (ML) tree inferred from the GBSSI-1b/1c sequences of Rubus. Bootstrap values $>50$ based on 1000 replicates are provided below the branches 
macilentus, $R$. simplex of subsect. Pungentes formed subclade I (0.69 PP). Subclade F included taxa from sects. Chamaebatus, Cylactis, and $R$. pentagonus of subsect. Alpestres from sect. Idaeobatus, as well as six taxa of subsect. Moluccani from sect. Malachobatus. Wellsupported (100\% BS, 1.00 PP) subclade J was composed of most sect. Malachobatus taxa with GBSSI-1b and three taxa with GBSSI-1c, which was almost consistent with group B1 (Fig. 4).

\section{Phylogenetic network}

A neighborNet diagram (Fig. 6) showed the same general patterns as the phylogenetic tree, corresponding to GBSSI-1a and GBSSI-1b/1c of the GBSSI-1 sequences in the two splits. The GBSSI-1a sequences could distinguish four broad groups: group A (corresponding to the major sect. Idaeobatus subclade in Fig. 4), group B (corresponding to sects. Malachobatus (Dalibardastrum + subsect. Peltati) - Cylactis - Chamaebatus - subsect. Alpestres subclade), group C (minor IdaeobatusCylactis subclade), and Anoplobatus group D. GBSSI-1b was occupied by species of the lineages E-J in Fig. 5.

\section{Discussion}

\section{Orthologs of GBSSI-1 gene in Rubus}

Orthology assessment is an important concern when using nuclear genes to reconstruct phylogeny, since paralogous sequences may lead to erroneous phylogenetic inferences [34, 35]. We carried out sequence alignment and phylogenetic analysis to test the orthology and paralogy of GBSSI-1. Rousseau-Gueutin et al. [26] hypothesized orthology of the DHAR sequences because they shared similar positions in both diploid and the cultivated octoploid strawberry genomes. The GBSSI-1 sequences from Rubus shared the same location among different genomes (Additional file 3). From the phylogenetic analysis (Fig. 2), we observed the Rubus sequences belonged to the same gene copy, GBSSI-1, which supported their orthologous status.

Compared with single copy GBSSI-2 in Rubus [25], GBSSI-1 gene was complex within the genus. Either GBSSI-1a or GBSSI-1b was detected in most diploids, while both of them were detected in $R$. pentagonus and $R$. peltatus, indicating their probable interspecific hybrid origin. Interestingly, different orthologs were identified based on gene structure within subsect. Corchorifolii of



Fig. 6 Neighbor-Net diagram based on uncorrected-P distances of GBSSI-1 DNA sequences of Rubus. The wavy lines represent the discrimination of two main sequences of GBSSI-1 gene. Major branches indicated $>90$ bootstrap values (data not shown). The capital letters correspond to the lineages of Figs. 4 and 5 
sect. Idaeobatus (Fig. 1, Additional file 2). Four taxa had GBSSI-1a and the other three had GBSSI-1b, which were clustered into subclades E and H1, respectively (Fig. 5). The two subclades belonged to clade II in gene trees, incongruent with their structure difference. We speculated that the GBSSI-1b originated from GBSSI-1a in some diploids by mutation. The two homoeologs also existed in majority of polyploids of sects. Malachobatus, Dalibardastrum, Chamaebatus, and Cylactis (unknown ploidy levels). Several sect. Malachobatus species even had GBSSI-1a, GBSSI-1b and GBSSI-1c. Tetraploid $R$. crassifolius (sect. Malachobatus) and blackberry cultivar 'Arapaho' (sect. Rubus) were exceptions with just one copy.

Of the 195 GBSSI-1 sequences in this study, seven contained stop codons and might have become pseudogenes, containing GBSSI-1a in $R$. fragarioides var. pubescens, GBSSI-1b in R. lambertianus and five GBSSI-1c sequences in $R$. lambertianus, $R$. lambertianus var. paykouangensis and $R$. panduratus (Additional file 2). All of them had deletions or insertions in the exon regions, leading to the nonsense mutation. The five GBSSI-1c sequences, with the missing fifth intron, might have become pseudogenes, but they might raise in quite recent since they had not yet led to long branches (the brief phylogram in the upper left corner in Fig. 5). Phylogenetic tree revealed that GBSSI-1c sequences were nested within GBSSI-1b clade (Fig. 5). It was reasonable to conclude that the GBSSI-1c type was directly originated from GBSSI-1b by mutation. Intron losses had been found in GBSSI-1 genes of diverse taxa, like subfamily Maloideae [29, 31] and Pooideae [36]. In some species of Poeae, the GBSSI intron loss was interpreted as a nonhomoplasious synapomorphy [36]. $\mathrm{Hu}$ [37] proposed the 'intron exclusion hypothesis', which suggested that a single intron could be precisely removed by double strand breaks (DSB) from a multiple-intron gene. This model of intron loss may explain the present results.

\section{Incongruence between GBSSI-1-based phylogeny and traditional Rubus classification}

Overall, GBSSI-1-based phylogeny largely supported Yü's rather than Focke's taxonomy. The results also generated some conflicts with the traditional morphology-based taxonomy, consistent with our previous study by chloroplast and single copy nuclear genes [25]. These incongruences probably suggested the need for a taxonomic revision using modern approaches.

The taxonomic treatments of $R$. ellipticus, $R$. ellipticus var. obcordatus, and $R$. pinfaensis have long been fraught with controversy. The dispute has mainly focused on two aspects, whether $R$. ellipticus and $R$. pinfaensis should be combined or not, and R. ellipticus var. obcordatus should be treated as a species $R$. obcordatus or a variety of $R$. ellipticus [2, 5, 6, 38-40]. In terms of character differences, $R$. ellipticus has dense pubescentia in blade back and $R$. pinfaensis has sparse villus [5]. On the contrary, the differences between $R$. ellipticus and $R$. ellipticus var. obcordatus not only focus on the leaflet shape and size, but also on the growth habits and habitat, inflorescence and flowering time [39]. Moreover, significant differences also exhibited in the pollen features, rDNA chromosomal distribution and genomic relationships by molecular cytogenetics [12, 39, 40]. In this study, three $R$. pinfaensis samples formed a strongly supported clade with the cluster of $R$. ellipticus and $R$. ellipticus var. obcordatus. The clade revealed obvious genetic divergence with any other species from both subsects. Stimulantes and Pungentes (Fig. 5, Additional file 5). Therefore, we supported to place them into a separate series Elliptici, sect. Idaeanthi, subg. Idaeobatus, as Focke proposed [2].

Rubus simplex was firstly placed into series Saxatiles of subg. Cylactis by Focke [1], while Yü et al. [5] and Lu \& Boufford [6] moved it into subsect. Pungentes of sect. Idaeobatus because its stipules adnate to base of petioles. Our phylogenies revealed that $R$. simplex formed a cluster with $R$. macilentus of sect. Idaeobatus rather than with sect. Cylactis species (Fig. 5, Additional file 5), partly supporting the traditional taxonomic treatment by Yü et al. and $\mathrm{Lu}[5,6]$. However, this cluster formed a clade with $R$. columellaris of subsect. Leucanthi, which exhibited deep divergence with other species of subsect. Pungentes (Fig. 4, Additional file 5). Thus, subsect. Pungentes was clearly demonstrated to be polyphyletic.

Rubus peltatus $(2 n=2 x=14)$ possesses some unique characters, such as peltate simple leaves, ovate stipules and 1-flowered with $5 \mathrm{~cm}$ or more in diameter, but distinct from other species of sect. Idaeobatus [5, 6, 41]. Both Species Ruborum [1] and Flora of China [5, 6] separately assigned it into subsect. Peltati of sect. Idaeobatus. Rubus peltatus revealed GBSSI-1a and $-1 \mathrm{~b}$ alleles, congruent with most tetraploid Malachobatus species. Here, it formed a moderately supported clade with some subsect. Moluccani species of sect. Malachobatus (Figs. 4, 5, 6). This suggested that $R$. peltatus might be closely related to polyploids. Moreover, diploid species of $R$. fulvus, $R$. micropetalus, and $R$. paniculatus have been reported to occur in the predominantly polyploid sect. Malachobatus [42-44]. Its rational taxonomic position needs to be explored further by multiple researches.

\section{Allopolyploid origin of Rubus polyploids}

Hybridization is believed to play an important role in plant speciation and evolution [24]. Chromosome numbers provide preliminary evidence for the possible hybrid origin of the sect. Malachobatus. The majority of the species from the sect. Idaeobatus present the chromosome 
number of $2 n=2 x=14$ [9]. On the other hand, species in the sects. Malachobatus, Dalibardastrum and Chamaebatus have been reported to have higher ploidy levels (e.g., $2 n=4 x=28$ for most species; $R$. amphidasys, $2 n=6 x=42$; R. buergeri, $2 n=8 x=56$ ) [9]. It is predicted that many speciation events in Rubus are associated with a change in ploidy levels. Thus, polyploidization may have played an important evolutionary role in the origin of the three sections. This study further offered the potential for new insights into the allopolyploid origin, especially in sect. Malachobatus.

\section{Section Malachobatus}

In our previous studies, bivalent pairing was the most predominant form in meiotic configuration, with just very few multivalents in some Malachobatus polyploids [13]. Moreover, polymorphism of 45S rDNA signal intensities by FISH were detected among them, implying different repeat copy numbers among different rDNA sites [12]. These results suggested that some sect. Malachobatus species be probable of allopolyploid origin. Here, GBSSI-1 homoeologs from the same polyploid individual dispersed in different well-supported clades in the GBSSI-1 gene tree (Figs. 4, 5, 6, Additional file 5), and some of these homoeologs were more closely related to homoeologs in other species than they were to each other, indicating that the homoeologs were donated by different ancestral taxa. As Wendel \& Doyle [45] and Fortune [46] proposed, the sequences duplicated by polyploidy should be each other's closest clades in autopolyploids, whereas be distributed in different clades in allopolyploids. This mechanism has been clearly illustrated in the origin of allotetraploid rice by Ge et al. [47]. Therefore, our findings provided strong evidence for allopolyploid origin of most sect. Malachobatus species. This hypothesis indicated that two kinds of diploids hybridized to form most allotetraploid species.

\section{Section Dalibardastrum}

Section Dalibardastrum species are also allopolyploids because of the co-occurrence of GBSSI-1a and $-1 \mathrm{~b}$ homoeologs. Rubus tsangorum and $R$. amphidasys share some morphological similarities, such as weak, densely bristly, prostrate stems, simple leaves, and terminal or axillary inflorescences, subracemes with 5 to 15 flowers, whereas they were reported as a tetraploid and hexaploid, respectively [9]. Both of them were strongly nested within sect. Malachobatus group (Figs. 4, 5, 6, Additional file 5), which suggested that they share parental ancestors from sect. Malachobatus. In addition, no other homoeologs besides GBSSI-1a and -1b were found in the hexaploid. As a consequence, the hexaploid might be derived from tetraploid without further hybridization, but only through unreduced gamete of tetraploid (4x and $2 x)$.

\section{Section Cylactis}

Members of sect. Cylactis formed a clearly polyphyletic group (Figs. 4, 5, 6, Additional file 5). They are creeping herbs with 3- or 5-foliolate compound leaves and several flowers in clusters or solitary [6]. This section contains various ploidy levels with diploid, tetraploid, and mixoploid [9]. Unfortunately, chromosome numbers of the examined taxa have never been reported. They all have two alleles of the GBSSI-1 gene, suggesting that hybridization events may have been involved in the origin. Specifically in sect. Cylactis, apomixis has also been found [48], hence various ploidy levels may be generated.

\section{The role of diploid sect. Idaeobatus in the evolution within Rubus}

Diploid sect. Idaeobatus is one of the largest sections in Rubus, which has been resolved as a polyphyletic group with several different evolutionary routes [25]. Here, GBSSI-1-based phylogeny strongly support our previous results (Figs. 4, 5, 6, Additional file 5). This was congruent with its morphological diversity [5, 6]. The majority of diploids with GBSSI-1a are composed of imparipinnately 3-9(-11)-foliolate leaves and flowers in mainly corymbs, while subsect. Corchorifolii with GBSSI-1a consist of simple leaves in 1-flowered, and the remaining diploids with GBSSI-1b with imparipinnately 3-5(-9)foliolate or simple leaves and flowers in subracemes. Particularly, $R$. pentagonus and $R$. peltatus with both GBSSI-1a and $-1 \mathrm{~b}$ is solitary flower with relative large diameter, with palmately 3 -foliate and simple leaves, respectively. Furthermore, Idaeobatus species exhibit both sexual and asexual reproduction, and some species could freely hybridize with each other and produce fertile offspring $[15-17,19]$. This probably contribute to the formation of new species, among which polyploids are contained.

Based on the structure difference and phylogeny, GBSSI-1b originated from GBSSI-1a in some diploids by mutation, then polyploidization happened between species with GBSSI-1a and -1b. Therefore, to some extent, the early-divergent diploid species with GBSSI-1a or -1b emerged before polyploid formation in the evolution of Rubus. Then they probably experience their own distinct evolutionary history with various evolutionary rates [25]. During the process, various but common diploidization events might occur in these polyploids [24], hence the allotetraploid is the most frequent and stable form within Rubus [9].

\section{Conclusions}

This study presented phylogenies of genus Rubus based on low-copy nuclear GBSSI-1 gene with a comprehensive taxon sampling with 140 Rubus individuals representing 102 taxa in 17 (out of the total 24) subsections 
of 7 (total of 12) sections at different ploidy levels. Either GBSSI-1a or GBSSI-1b was detected in most diploids (except for $R$. pentagonus and $R$. peltatus with both two alleles) of sect. Idaeobatus and blackberry cultivar of sect. Rubus. Both homoeologs (1a and 1b) were observed in majority of polyploids from sect. Malachobatus, as well as in sects. Dalibardastrum, Chamaebatus, and Cylactis species. Phylogenetic trees showed two clades I and II, corresponding to GBSSI-1a, and GBSSI-1b/1c sequences. GBSSI-1 homoeologs from the same polyploid individual dispersed in different well-supported clades in the GBSSI-1 gene tree, and some of these homoeologs were more closely related to homoeologs in other species than they were to each other, indicating that the homoeologs were donated by different ancestral taxa. Based on the structure difference and phylogeny, GBSSI$1 \mathrm{~b}$ originated from GBSSI-1a in some diploids by mutation, then polyploidization happened between species with GBSSI-1a and -1b. Two kinds of early-divergent ancestral diploids hybridized to form most extent allotetraploid species. This study provided new insights into allopolyploid origin and evolution from diploid to polyploid within genus Rubus at the molecular phylogenetic level, consistent with the taxonomic treatment by Yü et al. and Lu.

\section{Methods}

\section{Taxa sampling}

The Rubus classification of this study follows the system used in recent floristic treatments by Yü et al. [5] and $\mathrm{Lu} \&$ Boufford [6], since the majority of species sampled here are native in China. In total, we sampled 139 Rubus individuals, of which 85 (representing 59 taxa) are from 11 subsections of sect. Idaeobatus, one from sect. Rubus, 47 (representing 36 taxa) from 6 out of 13 subsections of sect. Malachobatus, two from sect. Dalibardastrum, one from sect. Chamaebatus, and three from sect. Cylactis (Additional file 2). These samples, with confirmed ploidy level, include 68 diploids $(2 n=14)$, one triploid $(2 n=21), 37$ tetraploids $(2 n=28)$, three hexaploids $(2 n=42)$, and one octoploid $(2 n=56)$ (Additional file 2) [9-11, 49-51]. Voucher specimens were deposited in the herbarium for horticultural plants, Sichuan Agricultural University (This herbarium is not indexed). Rubus odoratus $(2 n=14)$ [49] of subgenus Anoplobatus (almost corresponding to section by Yü) was also included in this study. Some representative species from family Rosaceae were selected as outgroups (Additional file 2).

\section{DNA isolation, amplification, cloning and sequencing}

Genomic DNA was extracted from silica-gel dried or frozen leaf tissues following the modified cetyltrimethyl ammonium bromide (CTAB) method [52]. Primers 3F (5'-TAC AAA CGA GGG GTT GAT CG-3') and 8R
(5'-GAT TCC AGC TTT CAT CCA GT-3') [30] were used to amplify GBSSI-1 gene. Primers 4F (5'-ACA AGA GGC AGC ATT AWA CAT CAG-3') and 4R (5'GGA AMC AAA AAG AGA GAA TCG GTA AGG-3') were designed here to sequencing the long 4th intron of GBSSI-1. The amplified fragment comprises $7 \mathrm{bp}$ at the 3 ' end of the third exon, four complete exons, five complete introns, and $7 \mathrm{bp}$ from the $5^{\prime}$ end of the eighth exon.

PCR amplification was performed in a PTC-200 thermocycler (Bio-rad, Hercules, CA). A volume of $25 \mu \mathrm{L}$ amplification mixture contains $20 \mathrm{ng}$ of template DNA, $2.5 \mu \mathrm{L}$ of $10 \times \mathrm{PCR}$ buffer $\left(10 \mathrm{mmol} \cdot \mathrm{L}^{-1} \mathrm{pH} 8.0\right.$ Tris$\left.\mathrm{HCl}, 50 \mathrm{mmol} \cdot \mathrm{L}^{-1} \mathrm{KCl}, 1.5 \mathrm{mmol} \cdot \mathrm{L}^{-1} \mathrm{EDTA}\right), 1.2 \mu \mathrm{L}$ of $\mathrm{MgCl}_{2}\left(25 \mathrm{mmol} \cdot \mathrm{L}^{-1}\right), 1.4 \mu \mathrm{L}$ of dNTP mix $\left(10 \mathrm{mmol} \cdot \mathrm{L}^{-}\right.$ $\left.{ }^{1}\right), 1 \mu \mathrm{L}$ of each primer $\left(5 \mu \mathrm{mol} \cdot \mathrm{L}^{-1}\right)$, and $1.5 \mathrm{U}$ of PfuDNA polymerase (Tiangen, Beijing). The cycling programme began with an initial pre-denaturation at $94{ }^{\circ} \mathrm{C}$ for $4 \mathrm{~min}$, followed by 30 cycles at $94{ }^{\circ} \mathrm{C}$ for $45 \mathrm{~s}$, $55^{\circ} \mathrm{C}$ for $1 \mathrm{~min}$ and $72^{\circ} \mathrm{C}$ for $1.5 \mathrm{~min}$. PCR finished after a final extension at $72^{\circ} \mathrm{C}$ for $20 \mathrm{~min}$.

PCR products were verified in a $1 \%$ agarose gel, and the target products were separated and purified by UNIQ-10 Column MicroDNA Gel Extraction Kit (Sangon, Shanghai, China). For diploids, purified products were directly sequenced with BigDye 3.1 reagents on an ABI PRISM 3730 automatic sequencer (Applied Biosystems, Foster City, California, USA) from both directions. Special attention was paid to those sites with overlapping peaks in the chromatograms, because they may indicate intra-individual variation (polymorphisms) [53]. If an obviously overlapping signal was detected in both the forward and reverse chromatograms, the site was considered to be putatively polymorphic between alleles or copies. Those products with polymorphic sites were cloned using TA cloning after Atailing and ligated to pMD20-T vector with a kit (Takara, Dalian, China). More than three clones per sample were se-

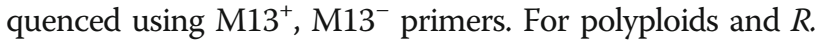
peltatus, $R$. pentagonus, two or more amplification bands were cloned separately to obtain sequences. All the sequences have been submitted to the GenBank database with accession numbers of MF595603-MF595796 (Additional file 2). In addition, GBSSI-1 sequences of $R$. odoratus and other Rosaceae species were downloaded from GenBank (Additional file 2) [29, 31, 54].

\section{Orthology identification}

To identify the orthology of GBSSI-1 gene sequences, we conducted gene sequence similarities and performed phylogenetic analysis. According to Yu's [32] definition of ortholog, the identity at the amino acid sequence level was employed by alignment with the reference genome of diploid $R$. occidentalis L. [33]. Sequence orthology analysis was also confirmed by phylogenetic analysis using exon sequences of the two GBSSI copies published 
from Rosaceae [29] together with corresponding sequences generated in this study from diploid Rubus. Sequences from Pisum sativum [55] and Rhamnus catharticus [29] were used as outgroups.

\section{Phylogenetic analyses}

We used CLC Genomics Workbench v7.5 (CLC bio, Qiagen, Boston, MA) for sequence editing and assemblying. The boundaries between exons and introns were determined by aligning with GBSSI-1 sequence of $R$. odoratus [29] and preservations of the 'GT' and 'AG' at two ends of introns. Sequences were aligned with Muscle [56] and manually adjusted in the Molecular Evolutionary Genetics Analysis software (MEGA 7.0) [57] with gaps treated as missing data. Sequence variation within and between different homoeologs was calculated by MEGA 7.0.

The obtained sequences from all species were first blasted (BlastN) against the released Rubus occidentalis to confirm that they are derived from the same GBSSI-1 locus. For those species with two or more forms of amplicons, all cloned and sequenced sequences were included in multisequence alignment in MEGA (v7.0) to genotype the patterns. Since all sequences despite of various length exclusively hit the GBSSI-1 region, they were treated as different alleles from the same gene of GBSSI-1. Three major variants denoted as GBSSI-1a, GBSSI-1b, and GBSSI-1c were obtained and all analyzed in phylogeny reconstruction. If two or more homoeologs were detected in one species, all of them were included for this species. The best fitting substitution model for GBSSI-1 was determined with the Akaike Information Criterion (AIC) [58] using JModelTest v2.1.1 [59]. The maximum likelihood (ML) tree was conducted using IQ-TREE v1.4.2 $[60,61]$. One thousand regular bootstrap replicates were performed to obtain confidence values for the branches. Bayesian inference (BI) was performed with MrBayes v3.2.1 [62]. The Markov chains Monte Carlo (MCMC) algorithm was run for 6,000,000 generations with one cold and three heated chains, at sample frequency of 100 . The first 1,500,000 generations were discarded as burn-in. Clade posterior probabilities (PP) were calculated from the combined sets of trees. All tree visualizations and annotations were achieved with iTOL v3 (Interactive Tree Of Life) online tool [63].

Phylogenetic networks can reflect the conflicting evolutionary signals and highlight reticulate evolution. Here, a network was constructed for the GBSSI-1 dataset with SplitsTree 4.14.2, using a NeighborNet diagram based on uncorrected- $P$ distance matrix [64]. Bootstrap support was estimated with 1000 replicates.

\section{Additional files}

Additional file 1: Survey on the species number and ploidy levels of Rubus taxonomy. (DOCX $93 \mathrm{~kb}$ )

Additional file 2: List of studied Rubus taxa, herbarium information, ploidy level, locality, and GenBank accession numbers of GBSSI-1 variants, and outgroups from family Rosaceae in this study. (DOCX $130 \mathrm{~kb}$ )

Additional file 3: The identity and E-value in GBSSI-1 of Rubus species by alignment with reference genome of diploid R. occidentalis L. (DOCX $96 \mathrm{~kb}$ )

Additional file 4: The simplified ML tree corresponding to Figs. 3 and 4 in Rubus. (JPG $2328 \mathrm{~kb}$ )

Additional file 5: Bayesian Inference (BI) tree inferred from the GBSSI-1 sequences of Rubus. Posterior probabilities $>0.50$ are shown below the branches. (JPG $7886 \mathrm{~kb}$ )

\section{Abbreviations}

BI: Bayesian inference; GBSSI: Granule-bound starch synthase I; LCNG: Low-copy nuclear gene; ML: Maximum likelihood

\section{Acknowledgements}

We greatly appreciated Prof. Daniel Potter at UC Davis and three anonymous reviewers for their valuable suggestions and comments for this manuscript. We also thank Li Zhang and Yin Liu for their great help in collecting samples.

\section{Authors' contributions}

XW conceived the project. YW and LL collected samples. YW and QC designed the research. YW performed the experiments. YW, QC, TC, JZ and WH carried out molecular phylogenetic analyses. YW and QC drafted the manuscript. HT, YL, YZ, BS and XW revised the manuscript. All authors have read and approved the manuscript for publication.

\section{Authors' information}

Yan Wang and Qing Chen are the co-author for this manuscript.

\section{Funding}

This work was supported by the National Natural Science Foundation of China (NSFC Grant Nos. 31272134, 31460206, 31600232, and 31672114).

\section{Availability of data and materials}

The data sets supporting the conclusions of this article are included within its additional files.

Ethics approval and consent to participate

Not applicable.

Consent for publication

Not applicable.

\section{Competing interests}

The authors declare that they have no competing interests.

\section{Author details}

${ }^{1}$ Institute of Pomology and Olericulture, Sichuan Agricultural University, Chengdu, China. ${ }^{2}$ College of Horticulture, Sichuan Agricultural University, Chengdu, China. ${ }^{3}$ Xizang Agriculture and Animal Husbandry College, Linzhi, China.

Received: 14 August 2018 Accepted: 2 July 2019

Published online: 10 July 2019

\section{References}

1. Focke WO. In: Stuttgart E, editor. Species Ruborum. Monographiae generis Rubi Prodromus part I. New York: Schweizerbart; 1910. p. 1-120.

2. Focke WO. In: Stuttgart E, editor. Species Ruborum. Monographiae generis Rubi Prodromus part II. New York: Schweizerbart; 1911. p. 121-223.

3. Focke WO. In: Stuttgart E, editor. Species Ruborum. Monographiae generis Rubi Prodromus part III. New York: Schweizerbart; 1914. p. 224-498.

4. Lu LD. A study on the genus Rubus of China. J Syst Evol. 1983;21:13-25. 
5. Yü DJ, Lu LD, Gu CZ, Guan KJ, Li CL. Rubus, Rosaceae. In: Board CaOSE, editor. Flora Reipublicae Popularis Sinicae. Beijing: Science Press; 1985. p. $10-218$

6. Lu LD, Boufford DE. Rubus Linnaeus, Sp. P1. 1: 492. 1753. In: Al-Shehbaz IA Bartholomew B, Boufford DE, Brach AR, Hong DY, Hu QM, Jeremie J, Kress WJ, Li DZ, Mcnamara WA, Peng Cl, Raven PH, Simpson DA, Turland NJ, Watson MF, Wu ZY, Xia B, Yang QE, Zhang LB, Zhang XC (eds.) Flora China. Beijing: Science Press; 2003. p. 195-285.

7. Kalkman C. The phylogeny of the Rosaceae. Bot J Linn Soc. 1988;98:321-41.

8. Alice LA, Campbell CS. Phylogeny of Rubus (Rosaceae) based on nuclear ribosomal DNA internal transcribed spacer region sequences. Am J Bot. 1999;86(1):81-97.

9. Thompson MM. Survey of chromosome numbers in Rubus (Rosaceae: Rosoideae). Ann Mo Bot Gard. 1997;84:128-64.

10. Naruhashi N, Iwatsubo Y, Peng Cl. Chromosome numbers in Rubus (Rosaceae) of Taiwan. Bot Bull Acad Sinica. 2002:43:193-201.

11. Wang XR, Tang HR, Duan J, Li L. A comparative study on karyotypes of 28 taxa in Rubus sect. Idaeobatus and sect. Malachobatus (Rosaceae) from China. J Syst Evol. 2008;46(4):505-15.

12. Wang Y, Wang XR, Chen Q, Zhang L, Tang HR, Luo Y, Liu ZJ. Phylogenetic insight into subgenera Idaeobatus and Malachobatus (Rubus, Rosaceae) inferring from ISH analysis. Mol Cytogenet. 2015;8:11.

13. Chen Q, Wang Y, Nan H, Zhang L, Tang HR, Wang XR. Meiotic configuration and rDNA distribution patterns in six Rubus taxa. Indian J Genet PI Br. 2015; 75(2):242-9.

14. Bammi R, Olmo H. Cytogenetics of Rubus. V. Natural hybridization between R. procerus PJ Muell. And R. laciniatus Willd. Evolution. 1966;20:617-33.

15. Iwatsubo Y, Naruhashi N. Karyomorphological and cytogenetical studies of Rubus parvifolius, R. coreanus and $R$. $\times$ hiraseanus (Rosaceae). Cytologia. 1991;56(1):151-6.

16. Iwatsubo Y, Naruhashi N. Cytotaxonomical studies of Rubus (Rosaceae): I. chromosome numbers of 20 species and 2 natural hybrids. J Jpn Bot. 1992; 67(5):270-5

17. Iwatsubo Y, Naruhashi N. Cytotaxonomical studies of Rubus (Rosaceae): II. Chromosome numbers of 21 species and 6 natural hybrids. J Jpn Bot. 1993a;68(3):159-65.

18. Iwatsubo $Y$, Naruhashi N. A comparative chromosome study of Rubus $X$ nikaii, R. parvifolius and R. phoenicolasius (Rosaceae). J Jpn Bot. 1996;71(6): 333-7.

19. Iwatsubo Y, Naruhashi N. Cytogenetic studies of natural hybrid, Rubus hiraseanus, and artificial hybrid between $R$. coreanus and R. parvifolius (Rosaceae). Cytologia. 1998;63(2):235-8.

20. Randell RA, Howarth DG, Morden CW. Genetic analysis of natural hybrids between endemic and alien Rubus (Rosaceae) species in Hawaii. Conserv Genet. 2004:5(2):217-30.

21. Mimura M, Mishima M, Lascoux M, Yahara T. Range shift and introgression of the rear and leading populations in two ecologically distinct Rubus species. BMC Evol Biol. 2014;14(1):209.

22. Iwatsubo Y, Naruhashi N. Cytogenetical study of Rubus $\times$ tawadanus (Rosaceae). Cytologia. 1993b;58(2):217-21.

23. Alice LA, Eriksson T, Eriksen B, Campbell CS. Hybridization and gene flow between distantly related species of Rubus (Rosaceae): evidence from nuclear ribosomal DNA internal transcribed spacer region sequences. Syst Bot. 2001;26(4):769-78.

24. Soltis DE, Soltis PS. The role of hybridization in plant speciation. Annu Rev Plant Biol. 2009;60:561-88.

25. Wang Y, Chen Q, Chen T, Tang HR, Liu L, Wang XR. Phylogenetic insights into Chinese Rubus (Rosaceae) from multiple chloroplast and nuclear DNAs. Front Plant Sci. 2016;7:968

26. Rousseau-Gueutin M, Gaston A, Aïnouche A, Aïnouche ML, Olbricht K, Staudt G, Richard L, Denoyes-Rothan B. Tracking the evolutionary history of polyploidy in Fragaria L.(strawberry): new insights from phylogenetic analyses of low-copy nuclear genes. Mol Phylogenet Evol. 2009;51(3):515-30.

27. Zimmer EA, Wen J. Reprint of: using nuclear gene data for plant phylogenetics: progress and prospects. Mol Phylogenet Evol. 2013;66: 539-50

28. Mason-Gamer RJ, Weil CF, Kellogg EA. Granule-bound starch synthase: structure, function, and phylogenetic utility. Mol Biol Evol. 1998;15(12):1658-73.

29. Evans RC, Alice LA, Campbell CS, Kellogg EA, Dickinson TA. The granulebound starch synthase (GBSSI) gene in the Rosaceae: multiple loci and phylogenetic utility. Mol Phylogenet Evol. 2000;17(3):388-400.
30. Michael K. Clarification of basal relationships in Rubus (Rosaceae) and the origin of Rubus chamaemorus. Bowling Green: Western Kentucky University; 2006.

31. Evans RC, Campbell CS. The origin of the apple subfamily (Maloideae; Rosaceae) is clarified by DNA sequence data from duplicated GBSSI genes. Am J Bot. 2002;89(9):1478-84.

32. Yu H, Luscombe M, L H, Zhu X, Xia Y, Han H, Bertin N, Chung S, Vidal M, Gerstein M. Annotation transfer between genomes: protein-protein interologs and protein-DNA regulogs. Genome Res. 2004;14:1107-18.

33. Jibran R, Dzierzon H, Bassil N, Bushakra JM, Edger PP, Sullivan S, Finn CE, Dossett M, Vining KJ, Vanburen R, Mockler TC, Liachko I, Davies KM, Foster TM, Chagné D. Chromosome-scale scaffolding of the black raspberry (Rubus occidentalis L.) genome based on chromatin interaction data. Hortic Res. 2018;5:8.

34. Sang T. Utility of low-copy nuclear gene sequences in plant phylogenetics. Critl Rev Biochem Mol. 2002;37:121-47.

35. Small RL, Cronn RC, Wendel JF. Use of nuclear genes for phylogeny reconstruction in plants. Aust Syst Bot. 2004;17:145-70.

36. Davis JI, Soreng RJ. A preliminary phylogenetic analysis of the grass subfamily Pooideae (Poaceae), with attention to structural features of the plastid and nuclear genomes, including an intron loss in GBSSI. Aliso: A J Syst Evol Bot. 2007;23(1):335-48.

37. Hu K. Intron exclusion and the mystery of intron loss. FEBS Lett. 2006; 580(27):6361-5.

38. Van Thuan N. Floredu Cambodge, duLaos, etdu Vietnam. Fascicule 7: Rosaceae II (Rubus). Paris: Museum Nationald'Histoire Naturelle; 1968.

39. Wang XR, Tang HR, Zhang HW, Zhong BF, Xia WF, Liu Y. Karyotypic palynological, and RAPD study on 12 taxa from two subsections of section Idaeobatus in Rubus L. and taxonomic treatment of R. ellipticus, R. pinfaensis, and R. ellipticus var. obcordatus. Plant Syst Evol. 2009; 283(1-2):9-18.

40. Wang $Y$, Wang XR, Chen Q, Zhang L, Liu Y, Tang HR. In situ hybridization analysis and taxonomic treatments of Rubus ellipticus, R. pinfaensis and $R$. ellipticus var. obcordatus. Acta Hortic Sinica. 2014;41(5):841-50.

41. Thompson M, Zhao C. Chromosome numbers of Rubus species in Southwest China. Acta Hortic. 1993:352:493-502.

42. Malik C. Cytology of some Indian species of Rosaceae. Caryologia. 1965; 18(1):139-49.

43. Mehra P, Gill B, Mehta J, Sidhu S. Cytological investigations on the Indian Compositae. I. North—Indian taxa. Caryologia. 1965;18(1):35-68.

44. Subramanian D. Cytotaxonomic studies of south Indian Rosaceae. Cytologia. 1987:52(3):395-403.

45. Wendel JF, Doyle JJ. Phylogenetic incongruence: window into genome history and molecular evolution. In: Soltis D, Soltis P, Doyle JJ, editors. Molecular systematics of plants II. New-York: Chapman and Hall; 1998.

46. Fortune P, Schierenbeck K, Ainouche A, Jacquemin J, Wendel J, Aïnouche ML. Evolutionary dynamics of Waxy and the origin of hexaploid Spartina species (Poaceae). Mol Phylogenet Evol. 2007:43(3):1040-55.

47. Ge S, Sang T, Lu BR, Hong DY. Phylogeny of rice genomes with emphasis on origins of allotetraploid species. Proc Natl Acad Sci U S A. 1999;96(25): $14400-5$.

48. Czapik R. Embryological problems in Rubus L. In: Erdelska O, editor. Fettilization and embryogenesis in ovulated plants. Bratislava: Veda; 1983. p. 375-9.

49. Thompson MM. Chromosome numbers of Rubus species at the national clonal germplasm repository. HortSci. 1995;30(7):1447-52.

50. Amsellem L, Noyer J-L, Hossaert-Mckey M. Evidence for a switch in the reproductive biology of Rubus alceifolius (Rosaceae) towards apomixis, between its native range and its area of introduction. Am J Bot. 2001; 88(12):2243-51.

51. Meng R, Finn C. Determining ploidy level and nuclear DNA content in Rubus by flow cytometry. J Am Soc Hortic Sci. 2002;127(5):767-75.

52. Zhou YQ. Application on DNA molecular markers Technology in Plant Study. Beijing: Chemical Industry Press; 2005. p. 9-34.

53. Zhao L, Jiang XW, Zuo YJ, Liu XL, Chin SW, Haberle R, Potter D, Chang ZY, Wen J. Multiple events of allopolyploidy in the evolution of the racemose lineages in Prunus (Rosaceae) based on integrated evidence from nuclear and plastid data. PLoS One. 2016;11(6):e0157123.

54. Campbell C, Evans R, Morgan D, Dickinson T, Arsenault M. Phylogeny of subtribe Pyrinae (formerly the Maloideae, Rosaceae): limited resolution of a complex evolutionary history. Plant Syst Evol. 2007;266(1):119-45. 
55. Dry I, Smith A, Edwards A, Bhattacharyya M, Dunn P, Martin C. Characterization of CDNAs encoding two isoforms of granule-bound starch synthase which show differential expression in developing storage organs of pea and potato. Plant J. 1992;2:193-202.

56. Edgar RC. MUSCLE: multiple sequence alignment with high accuracy and high throughput. Nucl Acids Res. 2004;32(5):1792-7.

57. Kumar S, Stecher G, Tamura K. MEGA7: molecular evolutionary genetics analysis version 7.0 for bigger datasets. Mol Biol Evol. 2016;33(7):1870-4.

58. Akaike H. A new look at the statistical model identification. IEEE Trans Automat Control. 1974;19(6):716-23.

59. Darriba D, Taboada GL, Doallo R, Posada D. jModelTest 2: more models, new heuristics and parallel computing. Nat Methods. 2012;9(8):772.

60. Nguyen LT, Schmidt HA, Von Haeseler A, Minh BQ. IQ-TREE: a fast and effective stochastic algorithm for estimating maximum-likelihood phylogenies. Mol Biol Evol. 2015:32(1):268-74.

61. Chernomor O, Von Haeseler A, Minh BQ. Terrace aware data structure for phylogenomic inference from supermatrices. Syst Biol. 2016;65:997-1008.

62. Ronquist F, Teslenko M, Van Der Mark P, Ayres DL, Darling A, Höhna S, Larget B, Liu L, Suchard MA, Huelsenbeck JP. MrBayes 3.2: efficient Bayesian phylogenetic inference and model choice across a large model space. Syst Biol. 2012;61(3):539-42.

63. Letunic I, Bork P. Interactive tree of life (iTOL) v3: an online tool for the display and annotation of phylogenetic and other trees. Nucl Acids Res. 2016;44(W1):W242-5.

64. Huson DH, Bryant D. Application of phylogenetic networks in evolutionary studies. Mol Biol Evol. 2006;23(2):254-67.

\section{Publisher's Note}

Springer Nature remains neutral with regard to jurisdictional claims in published maps and institutional affiliations.

Ready to submit your research? Choose BMC and benefit from:

- fast, convenient online submission

- thorough peer review by experienced researchers in your field

- rapid publication on acceptance

- support for research data, including large and complex data types

- gold Open Access which fosters wider collaboration and increased citations

- maximum visibility for your research: over $100 \mathrm{M}$ website views per year

At $\mathrm{BMC}$, research is always in progress.

Learn more biomedcentral.com/submissions 